\title{
Autenticação Automática de Assinaturas Online: Investigando Alternativas de Representação
}

\author{
Cassia I. G. da Silva, Guilherme L. A. Mota, Alexandre Sztajnberg, AruquiaB. M. \\ Peixoto \\ Universidade do Estado do Rio de Janeiro - UERJ \\ Instituto de Matemática e Estatística - IME / Bacharelado em Ciência da Computação \\ cassiaisac@gmail.com, \{guimota, alexszt\}@ime.uerj.br, \\ aruquia@gmail.com
}

\begin{abstract}
Resumo. A autenticação de assinaturas de forma automática tem aplicação direta no reconhecimento de indivíduos e suas credenciais. Entretanto, seu uso em sistemas críticos ainda é restrito devido a taxa de erro relativamente alta das implementações disponíveis. Este artigo apresenta um estudo abragente de algumas técnicas e algoritmos utilizados em visão computacional, aplicados com algum sucesso em sistema de reconhecimento de assinaturas. Entre os algoritmos estudados destaca-se o Dynamic Time Warping, utilizado para alinhar e medir a dissimilaridade entre duas assinaturas. Cada etapa do estudo foi avaliada utilizando um processo de autenticação implementado no Matlab, considerando o método online para captura de assinaturas.
\end{abstract}

Palavras-chave: Assinatura Online, Dynamic Time Warping.

\begin{abstract}
Automatic signature authentication has imediate application on the recognition of an individual and his/her credentials. However it's use in critical systems is still restricted due to the relative-high error rates observed in current implementations. This paper presents a comprehensive study of some techiques and algoriths used in computer vision, which are applied with some sucess in signature recogniton systems. Among the studied algorithms, the Dynamic Time Warping is highlighted, which is used to align and measure the dissimilarity between two signatures. Each stage of the study was evaluated using an autentication process implemented in the software Matlab, considering the online acquisition method.
\end{abstract}

Keywords: Online Signature, Dynamic Time Warping.

\section{Introdução}

Em nossa sociedade, a assinatura é uma forma legalmente constituída de conferir validade a documentos. A assinatura é um tipo de padrão biométrico comportamental que, em muitas situações, é considerada tão definitiva quanto padrões biométricos físicos, como a impressão digital, por exemplo. 
No sistema de compensação bancário, a validade da assinatura de um cheque é um item importante a ser verificado. A insuficiência dos procedimentos adotados ainda hoje, contribui para que cheques com assinaturas falsificadas sejam importante fonte de prejuízo para o sistema bancário e para o comércio varejista. $\mathrm{O}$ reconhecimento da autenticidade de assinaturas por computador dado, portanto, seu potencial para a redução destes prejuízos, é um tema de grande interesse de pesquisa. Outras vantagens potenciais, como tornar os procedimentos mais ágeis, práticos e reprodutíveis, quando comparado à autenticação realizada por peritos, também contribuem para a relevância desta área de pesquisa.

O reconhecimento da autenticidade de uma assinatura é um problema complexo. Vários fatores contribuem para este fato: a complexidade objetiva inerente a problemas com tamanha variabilidade de padrões; mudanças na forma de assinar, ao longo do tempo, como tamanho, características estilísticas e, até mesmo, a abreviatura de parte do nome; e influência do meio e do estado psicológico do indivíduo. A despeito dessas variações, algumas características são preservadas, permitindo, ainda assim, com segurança, a afirmação ou a negação de sua autenticidade.

No âmbito da autenticação automática de assinaturas, dois métodos são utilizados para a obtenção dos dados: offline e online. No método offline, a assinatura é feita sobre papel que é, posteriormente, digitalizado. Por outro lado, no método online, a assinatura é diretamente obtida em um dispositivo de hardware chamado mesa digitalizadora. Podem ser enumeradas diversas vantagens desta forma de captura em relação ao offline. A superioridade decorre tanto da não propagação de erros, quanto da obtenção de informações impossíveis de se obter de outra forma, dentre as quais: a sequência dos pontos da assinatura e a velocidade com que o indivíduo assina. Por este motivo, este documento se dedica à investigação da abordagem online.

Neste trabalho, são investigadas e avaliadas algumas abordagens e mecanismos para a representação de assinaturas, combinadas com algumas técnicas matemáticas e de visão computacional para a avaliação, discriminação e autenticação de assinaturas, com foco no método online. Observa-se que o emprego de técnicas diferentes ou a variação de atributos dentro de uma técnica pode alterar o resultado de uma classificação positiva ou negativamente. O objetivo do estudo é compreender algumas técnicas utilizadas com sucesso na autenticação automática de assinaturas e propor caminhos para aprimorar a qualidade da classificação.

Como estratégia de estudo, foi selecionado o método apresentado em [Kholmatov \& Yanikoglu (2004), Khol2005], vencedor do First International Signature Verification Competition (1st SVC), tendo obtido Equal Error Rate em torno de 3\%, resultado superior a várias outras propostas. A partir desta base, foram realizadas modificações nas características extraídas das assinaturas, tendo em vista a análise da influência da escolha destes atributos na exatidão da classificação. Os resultados obtidos com a abordagem proposta foram comparados com aqueles obtidos por [Khol2005], indicando que existe uma margem tangível para aprimoramento da classificação a partir de novas propostas de representação.

O restante do artigo está estruturado da seguinte forma. Na Seção 2, são apresentados os fundamentos necessários à compreensão do tema. Na Seção 3, é introduzido o método proposto em [Kholmatov \& Yanikoglu (2004)], que foi utilizado 
como base para o presente estudo. Uma avaliação abrangente dos parâmetros é apresentada naSeção 4 em conjunto com uma proposta para a obtenção automática de alguns destes parâmetros. Em seguida, na Seção 5 uma proposta alternativa de estrutura de dados para representação de assinaturas é discutida, assim como a comparação com os resultados obtidos no trabalho de referência ([Khol2005]). Finalmente, na Seção 6 considerações finais e caminhos para a continuação da investigação são apontados.

\section{Fundamentos}

\subsection{Dynamic Time Warping}

O algoritmo Dynamic Time Warping (DTW) mede a dissimilaridade entre duas sequências, não necessariamente de mesmo comprimento. Os elementos de tais sequências, por sua vez, correspondem a vetores de largura fixa.

Durante o cálculo da medida de dissimilaridade, o DTW realiza também o alinhamento das sequências. O objetivo é encontrar o melhor alinhamento não-linear entre elas, tal que as distâncias parcial e final entre as sequências sejam mínimas.

Diversas variantes deste algoritmo podem ser encontradas na literatura ([Wollmer et al. (2009), Krawczyk (2005), Kholmatov \& Yanikoglu (2004)]). Nesta apresentação, é empregada a mesma implementação que em [Kholmatov \& Yanikoglu (2004, 2005)]. Em tal variante, a distância total entre duas assinaturas $S_{1}$ e $S_{2}$ é calculada por intermédio da matriz $C$, conforme define a Equação 1:

$$
C[i, j]=\min \left\{\begin{array}{l}
C[i-1, j]+\gamma, \\
C[i, j-1]+\gamma, \\
C[i-1, j-1]+\operatorname{Dist}\left(S_{1}[i], S_{2}[j]\right)
\end{array}\right.
$$

onde $i=1 \cdots n$, em que $n$ é o número de elementos na assinatura $S_{1}$ e $j=1 \cdots m$, em que $m$ é o número de elementos na assinatura $S_{2}$. Assim sendo, $S_{v}[k]$ denota o $k$-ésimo elemento da $v$-ésima assinatura e

$$
\operatorname{Dist}(x, y)=\left\{\begin{array}{lr}
0, & \text { se }\|x-y\|<\theta, \\
\|x-y\|-\theta, & \text { caso contrário }
\end{array}\right.
$$

sendo $\gamma$ correspondente à penalidade adicionada pela ocorrência de um ponto discrepante nas sequências e $\theta$ à tolerância na distância entre dois elementos em comparação. O resultado da aplicação do algoritmo DTW, $C\left[l\left(S_{1}\right), l\left(S_{2}\right)\right]$, dá o escore de dissimilaridade de duas assinaturas, onde $l$ corresponde ao comprimento da assinatura.

A escolha dos parâmetros $\gamma$ e $\theta$ é de grande importância para o desempenho do algoritmo. Segundo Krawczyk (2005], se $\gamma$ (também conhecido por gap cost) é 
selecionado como sendo um número muito grande, então o DTW tende a ser muito rígido, resultando em baixas taxas de falsos positivos, mas altas taxas de falsos negativos. Se $\gamma$ é muito baixo, falsificações são "entortadas" de forma a conseguir o melhor alinhamento com as assinaturas verdadeiras, logo muitas falsificações terão baixos escores. O que resulta em baixas taxas de falsos negativos, mas altas taxas de falsos positivos. Os autores esclarecem que a intuição por trás do $\gamma$ é que, se dois pontos estão próximos, em termos da distância euclidiana, o custo de suas incompatibilidades também deveria ser pequeno. Isto permite que o algoritmo ache soluções, onde, se pontos consecutivos são similares, então, esses pontos são alinhados sem que seja imposta uma sanção rigorosa. Por outro lado, se dois pontos são muito diferentes, uma pena elevada deve ser aplicada. Uma análise do comportamento destes parâmetros é apresentada ao longo do presente estudo.

Vale a pena ressaltar que, se duas assinaturas são idênticas, o alinhamento do DTW é feito pela diagonal principal. Sendo as distâncias euclidianas destes elementos $(i, i)$, situadas ao longo da diagonal principal, iguais a zero, o DTW retorna $C\left(l\left(S_{k}\right), l\left(S_{k}\right)\right)$ também igual a zero. Isto é representado pela Figura 1(a).

Já quando as duas assinaturas possuem pequenas discrepâncias, o DTW, caso os parâmetros $\gamma$ e $\theta$ estejam ajustados corretamente, tende a alinhá-las da melhor maneira possível, impondo assim um valor pequeno de distância entre as assinaturas examinadas. A Figura 1(b) ilustra este fato.

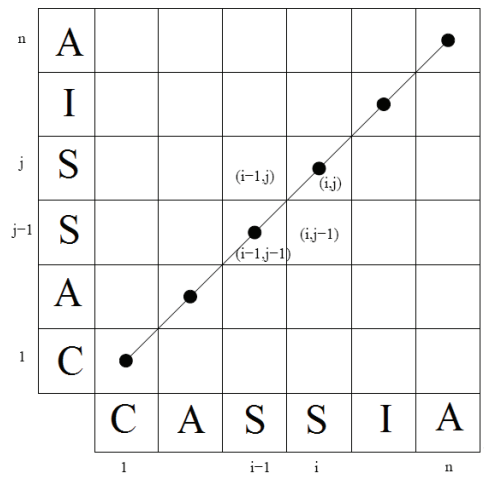

(a)

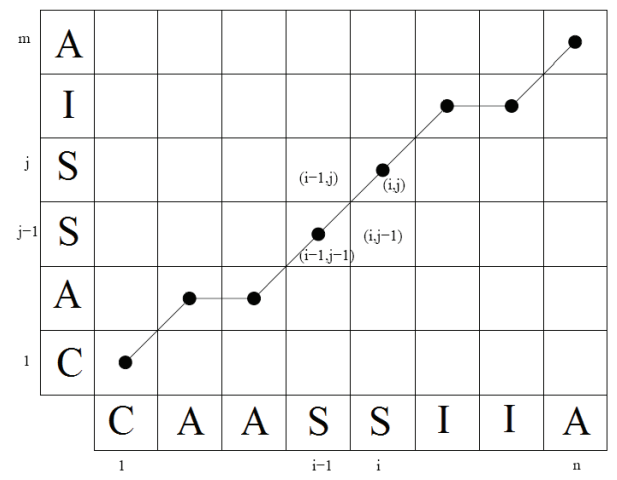

(b)

Figura 1. Alinhamento de pares de sequências com o DTW: (a) para assinaturas idênticas e (b) para assinaturas com poucas variações

\subsection{Análise dos Componentes Principais}

A Análise dos Componentes Principais (PCA) é um procedimento matemático que visa a obtenção de uma transformação ortogonal capaz de projetar o conjunto de dados no espaço ortonormal de maior variância, chamado espaço de componentes principais. Essa transformação é feita de maneira que a primeira componente principal possua a maior variância possível ([Duda et al. (1999), Johnson \& Wichern (1982)]).

Geralmente grande parte da variância dos dados é expressa por um número reduzido de componentes, sendo possível descartar as restantes sem perda significativa de informação. Tal redução pode ainda reduzir o número de parâmetros que devem ser estimados nas etapas seguintes, como por exemplo, na classificação. 
A base de componentes principais pode ser obtida a partir da matriz de covariância amostral dos dados observados. É equivalente aos autovetores da matriz de covariância, onde os respectivos autovalores expressam a variância observada em cada componente principal. $\mathrm{O}$ autovetor com o maior autovalor associado corresponde à componente principal do conjunto de dados usado. Isso significa que este é o relacionamento mais significativo entre as dimensões dos dados.

Este método foi utilizado nos trabalhos de referência ([Kholmatov \& Yanikoglu (2004, 2005)), para a redução de dimensionalidade dos dados de três para um. Para isto, os dados da distribuição são projetados na primeira componente principal, e assim, é feita sua classificação a partir classificador linear, descrito a seguir.

\subsection{Classificador Linear}

Classificadores devem ser capazes de distinguir as características em comum dentro de um grupo, para associar cada elemento ao seu respectivo grupo. Um classificador linear consegue isso, realizando uma decisão de classificação com base no valor de uma combinação linear das características.

Um classificador linear é provavelmente a técnica mais simples de aprendizagem de máquina. Para entender o problema, suponha duas classes e assuma que são linearmente separáveis por uma fronteira de decisão. O classificador visa a obtenção de um hiperplano que representa a fronteira de decisão. Assim, os objetos de classes diferentes podem ser separados por esta fronteira, [Duda et al. (1999)].

A etapa mais importante corresponde à definição dos parâmetros do plano que define a fronteira de decisão. Em muitas abordagens, esta tarefa é realizada em dois passos. Primeiramente, é obtido o vetor que representa a direção normal ao plano. Em seguida, é obtido um ponto do plano de decisão, [Duda et al. (1999)] e [Johnson \& Wichern (1982)].

\section{Trabalhos de Referência}

Os trabalhos tomados como base no presente estudo, [Kholmatov \& Yanikoglu (2004, 2005)], estão entre os mais bem sucedidos da literatura. As taxas de Equal Error Rate figuram entre as mais baixas para algumas das bases de dados empregadas em [GarciaSalicetti et al. (2009)]. Além disto, o sistema proposto pelos autores foi vencedor da First International Signature Verification Competition 2004 (sit (2004)).

A Figura 2 ilustra os principais passos seguidos nesta abordagem:

- Extração de atributos: extrair das assinaturas características que sejam relevantes à classificação.

- Alinhamento e cálculo de distância: comparação das características extraídas de duas assinaturas.

- Criação do perfil: é extraída, para cada usuário, a partir de um conjunto de assinaturas genuínas, uma série de informações que formam seu perfil.

- Normalização: as assinaturas a serem utilizadas nas etapas seguintes são normalizadas de acordo com o perfil do usuário com o qual é verificada a autenticidade. 
- Treinamento: obtém, a partir de um conjunto de assinaturas verdadeiras e falsificadas, a componente principal que será, posteriormente, utilizada na verificação das assinaturas.

- Verificação: uma vez treinado o sistema, uma assinatura qualquer cuja autenticidade é desconhecida pode ser verificada.

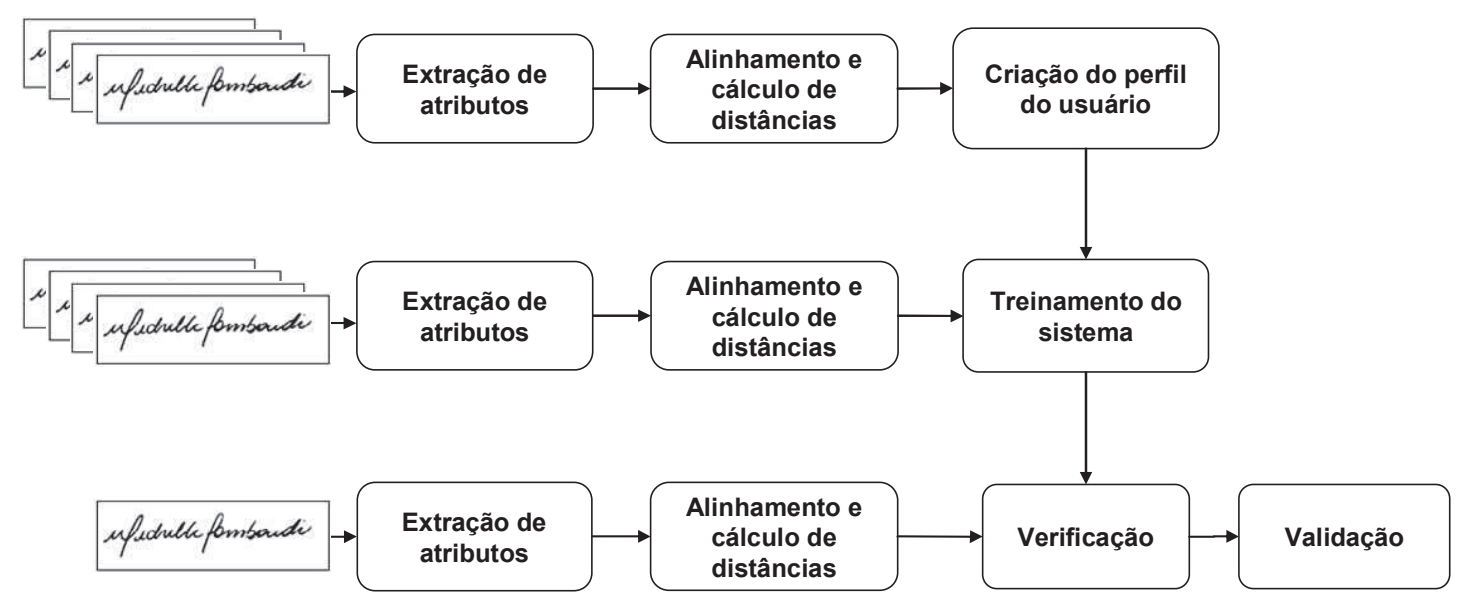

Figura 2. Fases para a autenticação de uma assinatura online no trabalho de referência

Nas seções que seguem, são descritas estas etapas assim como os resultados obtidos por este sistema.

\subsection{Extração de Atributos}

Kholmatov \& Yanikoglu (2004) ressaltam que, para que um sistema de autenticação de assinaturas apresente um bom desempenho, é preciso encontrar características que sejam tolerantes a pequenas mudanças dentro do conjunto de assinaturas genuínas. Por outro lado, além disto, tais medidas precisam acentuar a distinção de assinaturas falsificadas.

Os atributos utilizados são a diferença entre as coordenadas $x$ e $y$ de dois pontos consecutivos na trajetória da assinatura (que são chamados ao longo do texto de $\left(\Delta_{x}, \Delta_{y}\right)$ ). Deve ser mencionado que [Kholmatov \& Yanikoglu (2004)] avaliaram outros atributos, porém, dentre os testes feitos pelos autores, a diferença entre as coordenadas de dois pontos consecutivos possibilitaram os melhores resultados. Portanto, somente esta variante é empregada no presente estudo.

Kholmatov \& Yanikoglu (2004) também argumentam que um dos principais aspectos para o reconhecimento de uma falsificação nos sistemas de autenticação de assinaturas online é o tempo gasto para fazer a assinatura, uma vez que o tempo de duração da assinatura é muito mais difícil de ser reproduzido com precisão do que a forma da assinatura. Devido ao tempo ser uma característica implícita da captação dos pontos, os autores escolheram não fazer a reamostragem das assinaturas.

Para efetuar a comparação entre um par de assinaturas, é utilizado o algoritmo DTW, responsável pelo alinhamento e cálculo das distâncias. 


\subsection{Alinhamento e Cálculo das Distâncias}

$\mathrm{Na}$ aquisição da assinaturas online, o número de amostras nas sequências pode variar consideravelmente. Nestes casos, uma alternativa válida é o algoritmo DTW.

Como visto na Seção 2 são requeridos alguns parâmetros para que seja garantido um bom alinhamento entre as assinaturas e, assim, se obter uma melhor separação entre as classes genuínas e falsificadas. Em Kholmatov \& Yanikoglu $(2004,2005)$ não foram divulgados como foram obtidos tais parâmetros. Por este motivo, no presente estudo, será avaliada a influência destes parâmetros na classificação.

\subsection{Criação do Perfil}

Esta etapa consiste em tomar uma pequena amostra de assinaturas genuínas, chamada de conjunto de referência, para que seja extraído o perfil de assinatura do indivíduo. Em Kholmatov \& Yanikoglu (2005), foram fornecidas oito assinaturas no conjunto de referência. Essas assinaturas passam pela extração de atributos e em seguida, utilizando o DTW, são encontradas as distâncias entre cada par. A partir das distâncias calculadas entre todos os pares de assinaturas distintas, são extraídas três medidas utilizadas na criação do perfil do usuário:

- média das distâncias do conjunto de referência aos seus vizinhos mais próximos $\overline{D_{\min }}$;

- média das distâncias do conjunto de referência aos seus vizinhos mais distantes $\overline{D_{\max }}$;

- média das distâncias do conjunto de referência a um modelo (template) $\overline{D_{\text {template }}}$.

As duas primeiras médias são adquiridas da seguinte maneira: para uma assinatura base, toma-se a maior (ou menor) distância para as demais assinaturas do conjunto de referência. O processo é repetido utilizando-se como base todas assinaturas do conjunto de referência. Em seguida, é calculada a média dessas distâncias.

Nesta fase, é selecionada a assinatura modelo (ou template) que é utilizada nas outras fases do sistema. Esta é a assinatura do conjunto de referência que possui a menor média de distância entre todas as outras assinaturas deste conjunto.

\subsection{Normalização}

A partir desta etapa todas assinaturas apresentadas ao sistema são submetidas à normalização. Para cada usuário é obtido um vetor tridimensional onde os valores médios de distância, citadas anteriormente, representam o seu perfil. As medidas das distâncias máximas, mínimas às demais assinaturas e a distância média à assinatura modelo $\left(\overline{D_{\min }}, \overline{D_{\max }}, \overline{D_{\text {template }}}\right)$ são selecionadas para serem utilizadas na normalização dos valores obtidos nas próximas fases do sistema. $\mathrm{O}$ autor argumenta que normalizando essas distâncias através das médias correspondentes no conjunto de referência, eliminase a necessidade de calcular os limiares de decisão por usuário, sendo possível, assim, a utilização de um limiar global. 
Consequentemente, para cada assinatura do conjunto de treinamento é obtido um vetor $F_{y}$ que contém as distâncias obtidas no passo anterior normalizadas pelos valores obtidos na criação do perfil. O resultado é um vetor tridimensional $\left(F_{y}\right)$ obtido para cada assinatura, como mostra a Equação 3. Este vetor corresponde ao padrão que representa cada assinatura na classificação.

$$
F_{y}=\left[D_{\min } / \frac{}{D_{\min }}, D_{\max } / \frac{D_{\max }}{D_{\text {template }}} / \frac{}{D_{\text {template }}}\right]
$$

\subsection{Treinamento}

A fase de treinamento do sistema visa à obtenção do discriminante linear utilizado na classificação. Para o treinamento em Kholmatov \& Yanikoglu (2004), foram utilizadas 130 assinaturas no total, dentre as quais 76 são genuínas e 54 falsificadas. Os autores não mencionaram quantas assinaturas são utilizadas por usuário. Deve ser ressaltado que nesta etapa são empregadas assinaturas diferentes das assinaturas utilizadas durante a criação do perfil. Estas assinaturas são inseridas no sistema para que seja determinado o discriminante linear que será utilizado na distinção das assinaturas.

O treinamento consiste na obtenção da primeira componente principal fornecida pelo método de redução de dimensionalidade PCA, conforme exposto na Seção 2.2. Kholmatov \& Yanikoglu $(2004,2005)$ também empregaram o classificador Bayes para fornecer o discriminante. Como o desempenho do PCA foi superior, somente esta alternativa foi aplicada no presente estudo.

\subsection{Verificação}

Sendo $Y$ uma assinatura de teste pertencente ao conjunto de verificação e que não esteve presente nos conjuntos de criação do perfil e de treinamento. Após a obtenção do perfil e o treinamento, a assinatura de teste é submetida ao procedimento de extração de atributos normalizados $F_{y}$, apresentado na Equação 3. Então, o classificador, com auxílio do discriminante linear calculado no treinamento do sistema, é utilizado para determinar se $Y$ é uma assinatura genuína ou falsificada.

\subsection{Resultados}

Kholmatov \& Yanikoglu $(2004,2005)$ testaram três alternativas de representação da assinatura e dois classificadores, Bayes e linear. Os resultados observados na Tabela 1 foram adquiridos utilizando os atributos $\left(\Delta_{x}, \Delta_{y}\right)$ e o classificador linear.

Tabela 1. Resultados percentuais de erros do Classificador Linear

\begin{tabular}{|c|c|c|}
\hline Tipo & FRR (\%) & FAR (\%) \\
\hline Genuína & 1,65 & - \\
\hline Falsificação & - & 1,28 \\
\hline
\end{tabular}


A Tabela 1 apresenta o resultado do sistema utilizando o classificador Linear com a redução de dimensionalidade feita pelo PCA, onde obteve um erro de 1,65\% de falsas rejeições (FRR) e 1,28\% de falsas aceitações (FAR).

Deve ser ressaltado que para que o processo de alinhamento e cálculo de distâncias entre duas assinaturas seja bem sucedido são requeridos dois parâmetros, como descritos na Seção 2.1, expressos nas Equações 1 e 2. Como Kholmatov \& Yanikoglu $(2004,2005)$ não explicitam como tais parâmetros foram obtidos, a seguir, é apresentado um estudo mais aprofundado sobre os mesmos, tendo em vista a avaliação de sua influência na classificação das assinaturas.

\section{Estudo dos Parâmetros do DTW}

Nessa seção apresentam-se um conjunto de experimentos realizados para avaliar como estes parâmetros se comportam e como a escolha dos mesmos afeta o resultado da classificação de uma assinatura. Em seguida, é apresentada uma proposta para a escolha automática de valores para estes parâmetros e uma nova sequência de experimentos é realizada para verificar o seu desempenho.

\subsection{Visual Subcorpus}

Os experimentos realizados neste trabalho foram feitos com a base de dados SUSig (A. Kholmatov \& Yanikoglu (2008)), fornecida pelo próprio autor para a elaboração deste trabalho. A base de dados está dividida em duas partes: Visual e Blind Subcorpus. As assinaturas da Visual Subcorpus foram coletadas em um tablet sensível a pressão com tela LCD, onde os assinantes podiam ver suas assinaturas enquanto estavam assinando. Já os assinantes da Blind, não podiam ver essas imagens, o que resultou em falsificações mais pobres.

A Visual Subcorpus, utilizada em todos os experimentos descritos no presente trabalho, consiste de um banco de assinaturas doadas por 100 pessoas (29 mulheres e 71 homens). A maioria dos assinantes é estudante ou membro da Universidade de Sabancia, Turquia, com idades que variam entre 21 e 52 anos. Cada assinante forneceu 20 amostras de sua assinatura em duas diferentes sessões, 10 assinaturas em cada sessão. Cada pessoa, que forneceu sua assinatura, foi convidada a falsificar a assinatura de outra pessoa de forma aleatória. $\mathrm{O}$ falsificador teve a chance de visualizar a animação (velocidade e trajetória) da assinatura, várias vezes, e pôde praticar antes de falsificá-la. Deste modo, há um total de 10 falsificações, onde 5 são classificadas como habilidosas (FH) e 5 como altamente habilidosas (FAH), como mostra a Tabela 2.

Tabela 2. Sumário SUSig Visual Subcorpus

\begin{tabular}{|c|c|c|c|c|}
\hline Conjunto & Tipo & Usuários & Amostras/Usuários & Quantidade \\
\hline SEÇÃO 1 & Genuína & 100 & 10 & 1000 \\
SEÇÃO 2 & Genuína & 100 & 10 & 1000 \\
FH & Falsificação & 100 & 5 & 500 \\
FAH & Falsificação & 100 & 5 & 500 \\
Validação & Genuína/falsificação & 10 & $10 / 10$ & 200 \\
\hline
\end{tabular}




\subsection{Análise Experimental dos Parâmetros $\theta$ e $\gamma$}

Para avaliar a influência dos parâmetros $\theta$ e $\gamma$ foram desenvolvidos módulos em MATLAB®, versão 7.8.1 R2009a, reproduzindo o sistema descrito na Seção 3. Para este sistema foram submetidas para classificação assinaturas da Visual Subcorpus. Foram variados os parâmetros $\theta$ e $\gamma$, aplicados às Equações 1 e 2, registrando-se a taxa de erro (EER, do inglês Equal Error Rate) sobre as classificações das assinaturas. Para cada variação, o experimento foi repetido 3 vezes, e a média do ERR, então, calculada para cada valor atribuído a $\theta$.

Neste primeiro conjunto de experimentos foi utilizada a representação de assinaturas original que considera diferença entre dois pontos consecutivos $\left(\Delta_{x}, \Delta_{y}\right)$. Para cada usuário da base Visual Subcorpus, a seguinte configuração foi adotada: 8 assinaturas genuínas do conjunto para a criação do perfil do usuário (como em Kholmatov \& Yanikoglu (2004)), 4 assinaturas para o conjunto de treinamento (2 genuínas e 2 falsificadas) e 18 assinaturas para verificação, sendo 10 verdadeiras e 8 falsificadas.

Estes experimentos foram executados com os 20 primeiros usuários da base de dados. Os conjuntos para a criação do perfil, treinamento e verificação foram escolhidos de forma aleatória. Cada teste para 20 usuários durou em média 4h, em um computador com processador Pentium Dual Core, 3GB de memória RAM e 250GB de disco rígido.

O parâmetro $\theta$ foi variado de 1 a 1001 , com incremento de 50 unidades para cada teste, enquanto o parâmetro $\gamma$ foi variado de 1 a $61 \mathrm{com}$ passos de 20 . O gráfico da Figura 3 apresenta a média do EER para cada $\gamma$, com o respectivo desvio padrão para cada valor de $\theta$.

Pode ser observado na Figura 3 que a média de erro obtida tende a aumentar acompanhando o aumento do valor atribuído a $\theta$. Também pode ser verificado que o menor valor atribuído a $\theta(\theta=1)$ não corresponde ao menor erro encontrado, que foi obtido para $\theta=201$ e $\gamma=41$ com valor médio de erro (EER) igual a 2,7\%.

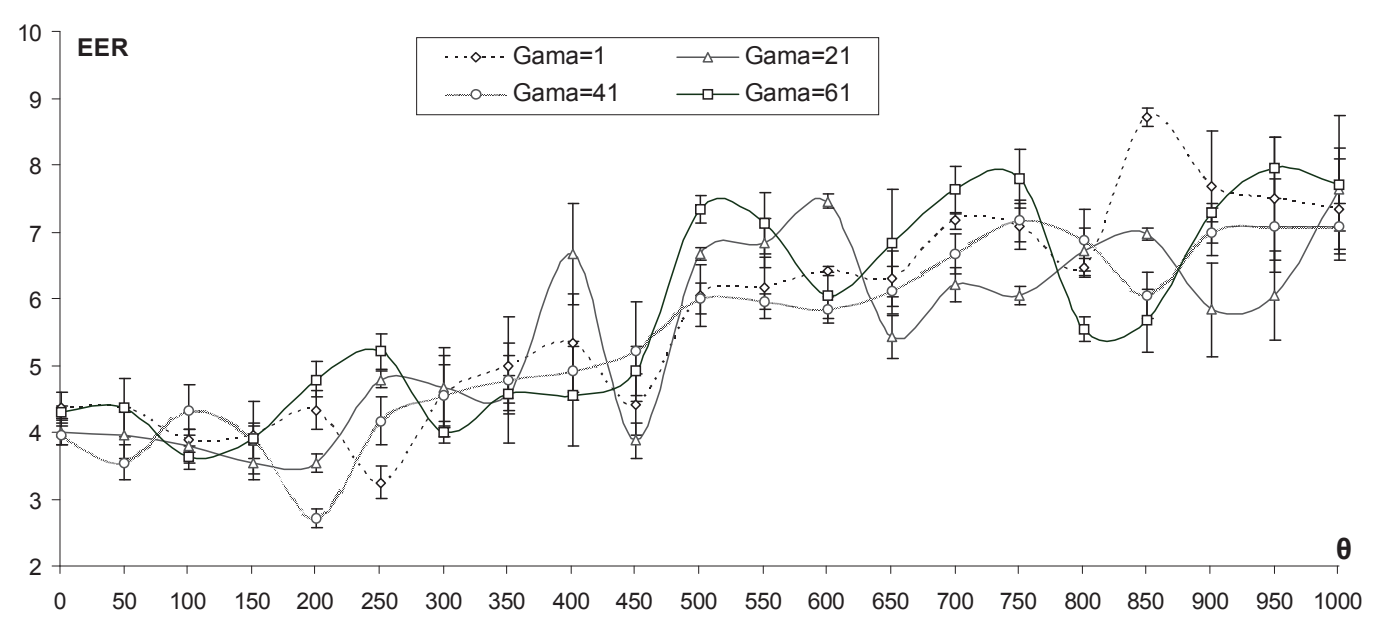

Figura 3. Variação da curva $y$ para vários valores de $\theta$ 
É observado também que a variação do parâmetro $\gamma$, neste intervalo, não influencia de forma significativa o resultado da classificação. Avaliadas as curvas de tendência (não apresentadas) deste parâmetro, as mesmas parecem coincidir.

Ressalta-se que se outra base de assinaturas fosse considerada, ou se as assinaturas fossem obtidas a partir de um tablet diferente, os valores das coordenadas dos pontos capturados também seriam diferentes. Assim, possivelmente, os valores para os parâmetros que alcançariam um desempenho ótimo, repetidos os experimentos com a nova base, provavelmente seriam outros.

Em um sistema real de autenticação não seria possível determinar valores de parâmetros repetindo-se testes para cada novo conjunto de assinaturas. Estes parâmetros teriam que ser determinados de forma prática e deveriam estar adaptados ao sistema, de modo a garantir um desempenho adequado.

\subsubsection{Cálculo de $\theta$}

Analisando isoladamente a condição dada pela Equação 2 pode-se observar que se $\theta$ for escolhido de muito maior do que as distâncias entre os pontos comparados de duas assinaturas, o valor atribuído à distância entre esses dois pontos será 0 . Ou seja, se estiver sendo comparada uma assinatura genuína e outra assinatura qualquer (podendo haver discrepância entre ambas), como $\theta$ tem um valor muito alto, (maior que a maior distância euclidiana entre os pontos dessas assinaturas) esta será classificada como uma assinatura verdadeira, pois a similaridade atribuída pelo DTW tenderá a ser baixa. Portanto, a escolha de valores demasiadamente grandes de $\theta$ pode levar a falsos positivos.

Em contrapartida, se $\theta$ for escolhido muito pequeno (menor do que a menor distância entre estas assinaturas) e se a comparação se der entre duas assinaturas do mesmo autor (poucas variações), o valor atribuído à distância da Equação 2 será $\|x-y\|-\theta$, que por consequência também será um valor alto. Portanto, possivelmente esta será classificada como uma assinatura falsa, pois a distância atribuída pelo DTW será muito alta. Consequentemente, a escolha de valores demasiadamente pequenos de $\theta$ pode levar a um numero grande de falsos negativos.

Considerando estas informações e com o objetivo de tornar automática a atribuição do valor de $\theta$, por um lado, e adaptado às assinaturas sob avaliação, por outro, é proposta a seguinte estratégia. Para cada par de assinaturas, calcula-se a matriz de distâncias $\operatorname{Dist}\left(S_{1}[i], S_{2}[j]\right)$, onde $i=1 \cdots n$ e $j=1 \cdots m$ são números de pontos dos vetores de atributos das assinaturas $S_{1}$ e $S_{2}$, respectivamente. Aqui também é utilizada a distância euclidiana. $\mathrm{O}$ valor atribuído ao parâmetro $\theta$ será a média da matriz de distâncias. Ou seja, o cálculo do parâmetro $\theta$, é dado pela equação

$$
\theta=\frac{1}{(n \times m)} \sum_{i=1}^{n} \sum_{j=1}^{m}\left|S_{1}[i]-S_{2}[j]\right|
$$

Esta proposta para o cálculo dinâmico de $\theta$ é inspecionada na próxima seção. 


\subsection{Análise dos casos extremos do parâmetro $\theta$}

Como primeira avaliação da proposta do cálculo dinâmico de $\theta$ foram experimentadas 3 abordagens para o uso das equações do DTW considerando 4 casos diferentes. Observando as condições de distâncias dadas pela Equação 2, foram testadas cada uma das condições em separado, ou seja, ao invés da equação do DTW ser descrita como visto nas Equações 1 e 2, foram analisados os casos específicos conforme apresentado nas equações da Figura 4: Abordagem I: $\theta$ é escolhido muito grande; Abordagem II: $\theta$ é escolhido muito pequeno; Abordagem III: $\theta$ calculado dinamicamente.

(a) Abordagem I: $C[i, j]=\min \left\{\begin{array}{l}C[i-1, j]+\gamma \\ C[i, j-1]+\gamma \\ C[i-1, j-1]+0\end{array}\right.$

(b) Abordagem II: $C[i, j]=\min \left\{\begin{array}{l}C[i-1, j]+\gamma, \\ C[i, j-1]+\gamma, \\ C[i-1, j-1]+|\|x-y\|-\theta|\end{array}\right.$

(c) Abordagem III: $C[i, j]=\min \left\{\begin{array}{l}C[i-1, j]+\gamma, \\ C[i, j-1]+\gamma, \\ C[i-1, j-1]+\operatorname{Dist}\left(S_{1}[i], S_{2}[j]\right)\end{array}\right.$ onde, $\operatorname{Dist}(x, y)=\left\{\begin{array}{lr}0, & \text { se }\|x-y\|<\theta, \\ \|x-y\|-\theta, & \text { caso contrário }\end{array}\right.$

Figura 4. Equações para o cálculo do DTW

Observe que as duas primeiras equações (a) e (b) da Figura 4, são derivações da condição dada pela Equação 2 (esta última equação representada na Figura 4(c)). Aqui, já está sendo considerado o parâmetro $\theta$ como sendo a média da matriz de distância e foi atribuído o valor $\gamma=2$, para o outro parâmetro.

Quatro situações são analisadas: uma assinatura comparada com ela mesma, idênticas; duas assinaturas de dois autores distintos, completamente diferentes (que pode ser classificada como uma falsificação aleatória); duas assinaturas do mesmo autor (mas diferentes entre si); e uma assinatura genuína com sua respectiva falsificação.

A superfície DTW para cada caso foi plotada da seguinte maneira: as coordenadas $x, y$ da superfície, representam a posição das coordenadas dos vetores de características das assinaturas $S_{1}$ e $S_{2}$ e $z$ a distância DTW entre eles. Ou seja, o ponto $(2,3, D)$ da superfície é a distância DTW, $D$, entre o segundo ponto do vetor de características da assinatura 1 com o terceiro ponto do vetor de características da assinatura 2. A cor azul escuro é determinada pelo valor mais baixo entre as distâncias. Já o maior valor é caracterizado pela cor vermelha. Ou seja, quanto mais avermelhado for o último ponto na matriz DTW maior é a distância entre as assinaturas. A Figura 5 
apresenta a visualização do plano $x y$ das superfícies plotadas para o caso de assinaturas idênticas para as três abordagens (as figuras para os demais casos são omitidas por restrições de espaço).

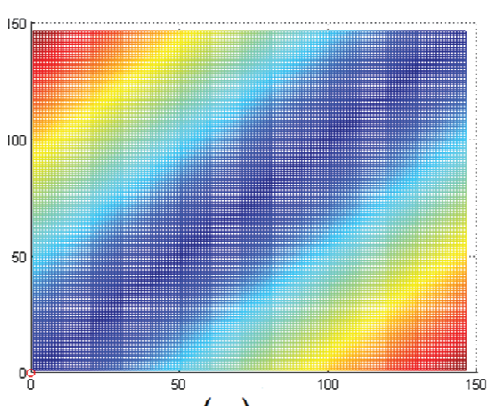

(a)

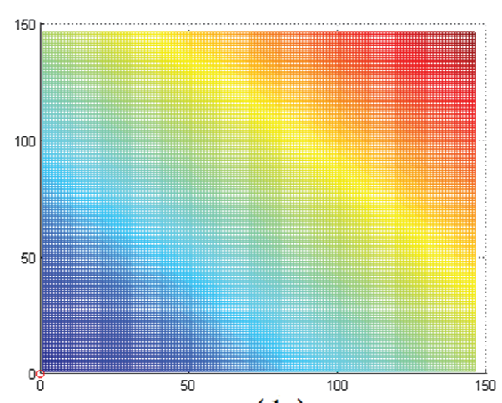

(b)

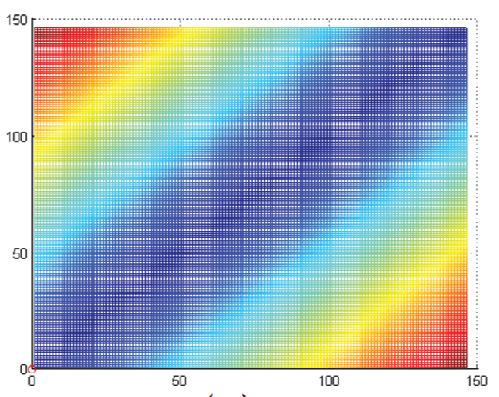

(c)

Figura 5. Visualização $x y$ das superfícies DTW para assinaturas idênticas

A Tabela 3 consolida os valores da distância DTW obtidas nas três abordagens para os quatro casos analisados. Observa-se que para todos os casos, a distância DTW tende a ser muito alta quando obedecemos rigorosamente a segunda condição da Equação 2, ou seja, quando $\operatorname{Dist}(x, y)=\|x-y\|-\theta$. O que ocasionaria rejeições em todos os casos, até mesmo considerando a mesma assinatura, na classificação do sistema.

Da mesma forma, utilizando apenas a primeira condição da Equação 2, ou seja, $\operatorname{Dist}(x, y)=0$, a distância DTW tende a ser muito pequena. Isso acarreta muitas falsas aceitações.

Utilizando a definição do DTW como visto em Kholmatov \& Yanikoglu (2004, 2005) e adotando o parâmetro $\theta$ como sendo a média da matriz de distâncias euclidianas, é possível verificar que bons resultados são obtidos nos quatro casos, exceto para o caso das assinaturas completamente diferentes. O que produziria uma taxa de erro um pouco maior.

Tabela 3. Distâncias DTW para as 3 abordagens e os 4 casos analisados

\begin{tabular}{|l|c|c|c|}
\hline Casos & Abordagem I & Abordagem II & Abordagem III \\
\hline Assinatura com ela mesma & 0 & 561 & 0 \\
\hline Assinaturas diferentes & 36 & 521 & 104 \\
\hline Assinatura com a falsificação & 632 & 2044 & 644 \\
\hline Assinaturas do mesmo autor & 2 & 578 & 10 \\
\hline
\end{tabular}

Esta primeira avaliação do comportamento do parâmetro $\theta$ calculado pela média das distâncias aponta a proposta como adequada para a aplicação de autenticação de assinaturas online. Dispõem-se agora de uma opção para a escolha automática deste parâmetro. 


\subsection{Avaliação da Proposta para Cálculo de $\theta$}

Para comprovar a utilidade da proposta, nesta seção novos experimentos são realizados para avaliar o desempenho do processo de autenticação considerando o valor do parâmetro $\theta$ calculado de forma dinâmica. Estes experimentos utilizaram basicamente a mesma configuração daqueles descritos na Seção 4.2.

Em princípio, na primeira bateria de testes não foi observada grande influência do parâmetro $\gamma$ sobre a classificação. Da mesma forma, os novos testes foram refeitos para avaliar se a influência exercida sobre a classificação pelo parâmetro $\gamma$ mudaria de configuração. Com isso, os experimentos foram refeitos variado-se este parâmetro dentro de um intervalo de valores consideráveis, mas desta vez o parâmetro $\theta$ calculado dinâmicamente pela nova proposta e não mais variado sistematicamente dentro de uma faixa.

O intervalo de variação de $\gamma$ foi ampliado em relação em relação à primeira bateria de testes, de 1 a 201, e foi diminuindo o intervalo do incremento, para 10 unidades a cada teste. Cada teste foi repetido 5 vezes, obtendo-se assim, a média de erro (EER) para cada ponto. A Figura 6 apresenta o resultado para cada valor atribuído a $\gamma$, assim como o desvio padrão para cada caso, calculado com um grau de confiança de 90\% segundo uma distribuição $t$ de Student.

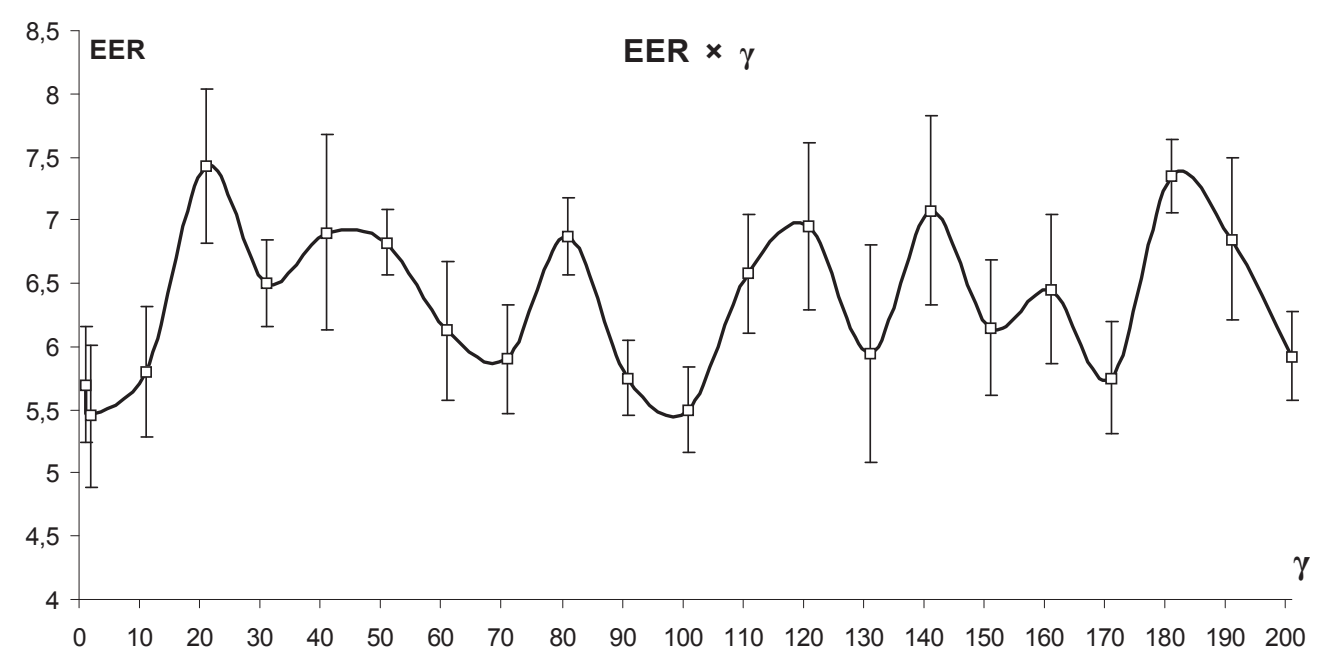

Figura 6. Variação do EER em função do parâmetro $\gamma$.

Pode ser verificado que foram obtidos algumas baixas taxas de erro ao longo desta variação e que dentre os valores atribuídos a $\gamma$, o que obteve menor percentual de erro foi o $\gamma=2$, com um desvio padrão de 1,525 , que obteve uma média de erro de $5,45 \%$.

Por outro lado, dentro da faixa de valores atribuídos à $\gamma$, os valores médios de EER obtidos na autenticação de assinaturas parecem não obedecer a um padrão de crescimento ou de decrescimento. Assim, decidiu-se não ampliar os testes em torno de $\gamma$ neste estágio.

Comparando-se os valores dos melhores resultados apresentados nos gráficos na Figura 3 e na Figura 6, pode-se considerar que mesmo com o aumento do EER de 
$2,71 \%$ para $5,45 \%$, a proposta pode compensar o inconveniente da busca exaustiva pelos valores ótimos dos parâmetros.

\section{Proposta de Representação de Assinaturas, Avaliação e Resultados}

Como passo sequente do trabalho é proposta uma forma alternativa para a representação de uma assinatura. A ideia central é incorporar à representação um pouco mais de informação sobre a forma da assinatura capturada aproximando um pouco mais o sistema automático de autenticação da maneira que um perito faria para reconhecer uma assinatura. A forma da assinatura é uma propriedade que, para a grafoscopia, é fundamental para indicar a autenticidade de uma assinatura, dado que cada pessoa possui uma forma única e pessoal de assinar.

A abordagem adotada é incluir a informação da curvatura dos pontos de uma assinaura para sua representação. O módulo de extração de atributos foi adaptado para incluir esta informação. Esta alternativa de representação é avaliada e comparada com representação original do trabalho de Kholmatov utilizada nos experimentos da seção anterior.

\subsection{Nova Representação e Extração de Atributos}

Com o objetivo de aprimorar o desempenho do sistema de autenticação é proposta a utilização da informação da variação da curvatura, considerando cada ponto adquirido pela mesa digitalizadora. É importante ressaltar que vetores normais indicam a variação da curvatura ao longo de uma curva, ou superfície. A expectativa era a de que a nova representação incluiria uma carga de informação maior sobre a assinatura, além de sua e que a mesma seria invariante à escala, rotação e translação, já que a curvatura de uma assinatura não apresentaria tantas variações.

A extração da informação da variação da curvatura é obtida da seguinte forma: dados três pontos consecutivos na trajetória da assinatura, são calculados os vetores que darão o sentido de orientação da escrita. Isto é, para o ponto $P_{i}$ é calculado o vetor $\overline{P_{i-1} P_{i+1}}$.

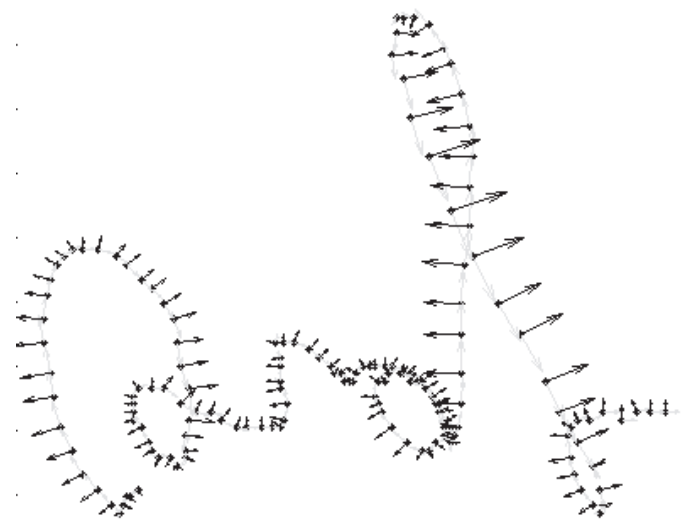

Figura 7. Vetores normais (escuros) aos vetores calculados sobre as coordenadas dos pontos (claros) coletados no tablet 
Logo após, em cada ponto $P_{i}$ é calculado o vetor normal aos calculados anteriormente, que resulta na variação da curvatura ao longo da trajetória da assinatura. Observe a Figura 7. Estes vetores são utilizados para representar a assinatura a ser classificada. O resultado obtido é uma matriz de ordem $n \times 2$, onde $n$ é numero de pontos capturados pelo tablet $\mathrm{e}$ as colunas correspondem às componentes $x y$ dos vetores encontrados.

Para a comparação entre duas assinaturas, cada uma delas é representada por um vetor de atributos. Os vetores são submetids ao DTW para que sejam alinhados e em seguida determinada a distância existente entre eles. Assim, o processo de autenticação segue como descrito na Seção 3. Os experimentos desta avaliação são discutidos a seguir.

\subsection{Experimentos}

Nesta nova rodada de experimentos, foram utilizados os primeiros 20 usuários da base de dados, sendo no treinamento empregado somente um único usuário. Para estes experimentos, foi variado o parâmetro $\gamma$ entre o intervalo de 1 a 201, com incremento de 10. O parâmetro $\theta$ foi calculado com a nova proposta seguindo a Equação 4. Cada teste foi repetido cinco vezes para a obtenção do EER médio para cada valor de $\gamma$. As configurações dos experimentos anteriores foram novamente usadas.

Vale ainda lembrar que apenas um usuário foi utilizado para o cálculo do PCA e que as falsificações utilizadas em nossos experimentos são classificadas como altamente habilidosas. Os mesmos experimentos foram repetidos para a extração de atributos original de Kholmatov \& Yanikoglu (2004) e para a forma proposta na seção anterior.

A Figura 8 mostra o gráfico da variação do parâmetro $\gamma$ e suas respectivas médias de erro percentual (EER) na classificação das assinaturas, obtidas em ambas as propostas e o desvio padrão calculado com um grau de confiança de $90 \%$ segundo uma distribuição $t$ de Student. A barra mais escura representa a implementação proposta por Kholmatov \& Yanikoglu $(2004,2005)$ com sua extração de atributos e a barra com mais clara representa a proposta de representação apresentada neste trabalho.

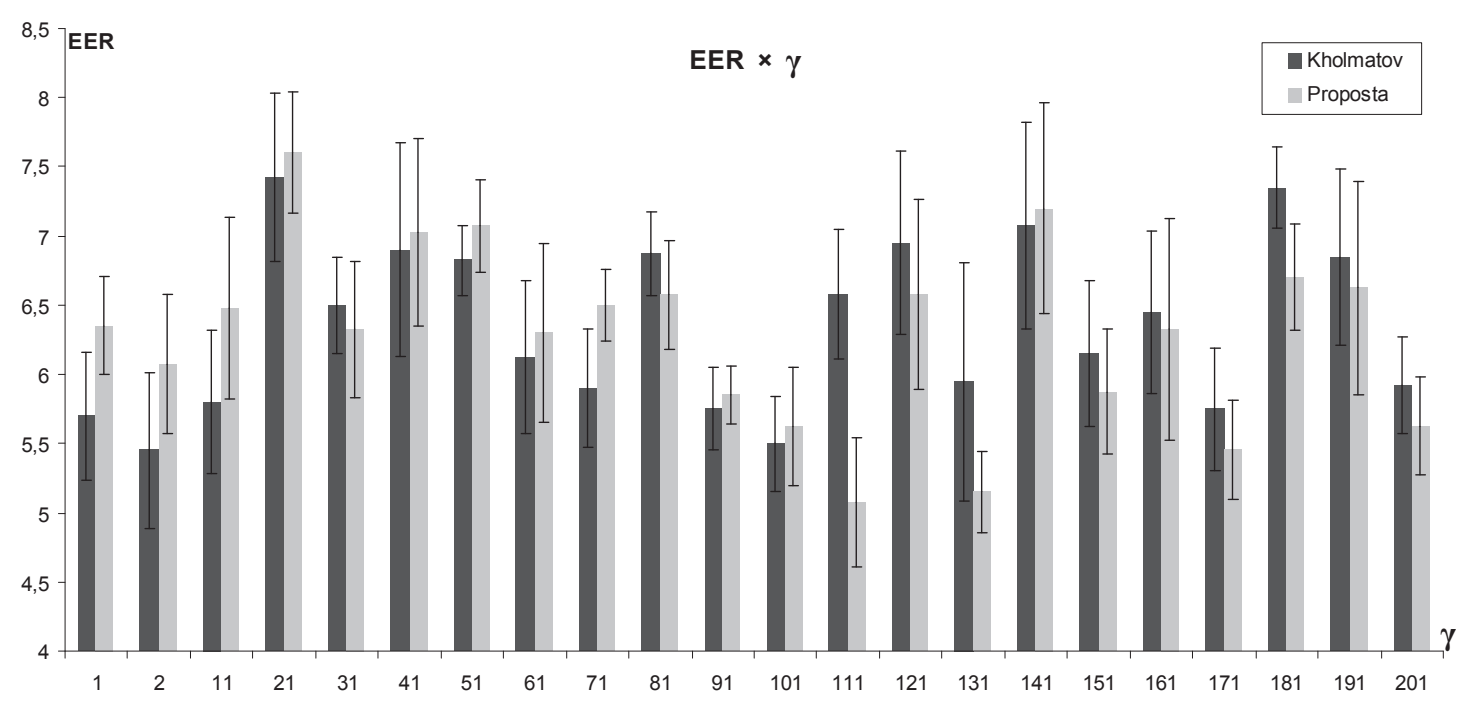

Figura 8. Média do EER em função do parâmetro $Y$ 
É possível observar que a menor média de erros foi obtida pela representação de atributos aqui proposta, para $\gamma=111$. Já para a extração de atributos utilizada por Kholmatov \& Yanikoglu (2004, 2005), com $\gamma=2$.

Observe que os resultados obtidos pela extração de atributos apresentada neste trabalho, em alguns casos, foram menores do que os obtidos pela extração de atributos sugeridos por Kholmatov \& Yanikoglu (2004). Pode ser analisado, também, que a menor média de erros obtida com a representação proposta, para $\gamma=111$ e $\gamma=131$, foram inferiores a todas as médias de erros obtidas pela proposta vista em Kholmatov \& Yanikoglu (2004).

Com base nestes resultados, estes últimos experimentos foram repetidos para $\gamma=111$ e $\gamma=2$, agora para todos os 94 usuários da base de dados Visual Subcorpus. Contudo, o usuário utilizado no treinamento (cálculo do PCA) foi diferente daquele utilizado nos experimentos anteriores.

Para $\gamma=2$ foi obtida uma média da taxa de erro de $3,78 \%$ de EER para a representação da assinatura proposta e discutida na Seção 5.1, e de 3,28\% de EER para a representação apresentada em Kholmatov \& Yanikoglu $(2004,2005),\left(\Delta_{x}, \Delta_{y}\right)$. Essas médias, assim como desvio padrão, foram obtidas pela repetição do experimento por 10 vezes. O sistema executa a implementação proposta por Kholmatov \& Yanikoglu (2004, 2005), apenas trocando o módulo de extração de atributos. Esses resultados são apresentados na Tabela 4.

Tabela 4. Médias de EER para ambas as representações, $\gamma=2$

\begin{tabular}{|l|l|l|}
\hline Extração de atributos & Kholmatov & Proposta \\
\hline Média & 3,28457 & 3,78191 \\
\hline Desvio Padrão & 0,54465 & 0,49658 \\
\hline
\end{tabular}

Para $\gamma=111$ os valores dos erros EER obtidos através desses experimentos são ligeiramente menores do que os obtidos pela proposta de Kholmatov \& Yanikoglu (2004) e também que a média de erros para a proposta deste trabalho mostrou-se sutilmente inferior quando calculada para $\gamma=2$. Observe também que para esse experimento, o valor do desvio padrão obtido para a representação da assinatura proposta aqui foi maior do que o obtido pela diferença entre dois pontos consecutivos, $\left(\Delta_{x}, \Delta_{y}\right)$. A Tabela 5 mostra as médias percentuais de erro dos resultados obtidos através de 10 rodadas, assim como o desvio padrão, para cada uma das propostas avaliadas.

Tabela 5. Médias de EER para ambas as representações, $\gamma=111$

\begin{tabular}{|l|l|l|}
\hline Extração de atributos & Kholmatov & Proposta \\
\hline Média & 3,52926 & 3,47872 \\
\hline Desvio Padrão & 0,59813 & 0,61443 \\
\hline
\end{tabular}




\subsubsection{Análise dos Resultados das Propostas}

O gráfico da Figura 9 apresenta as médias de erro obtidas por ambas as propostas para os dois valores de $\gamma$ (2 e 111), assim como o intervalo de confiança, calculado com um grau de confiança de $90 \%$ segundo uma distribuição $t$ de Student para cada situação. Estes resultados foram obtidos com os experimentos realizados com os 94 usuários da base de dados Visual Subcorpus, onde suas falsificações são classificadas como altamente habilidosas. A barra mais escura representa o resultado da implementação proposta por Kholmatov \& Yanikoglu $(2004,2005)$ e a mais clara a proposta sugerida aqui.

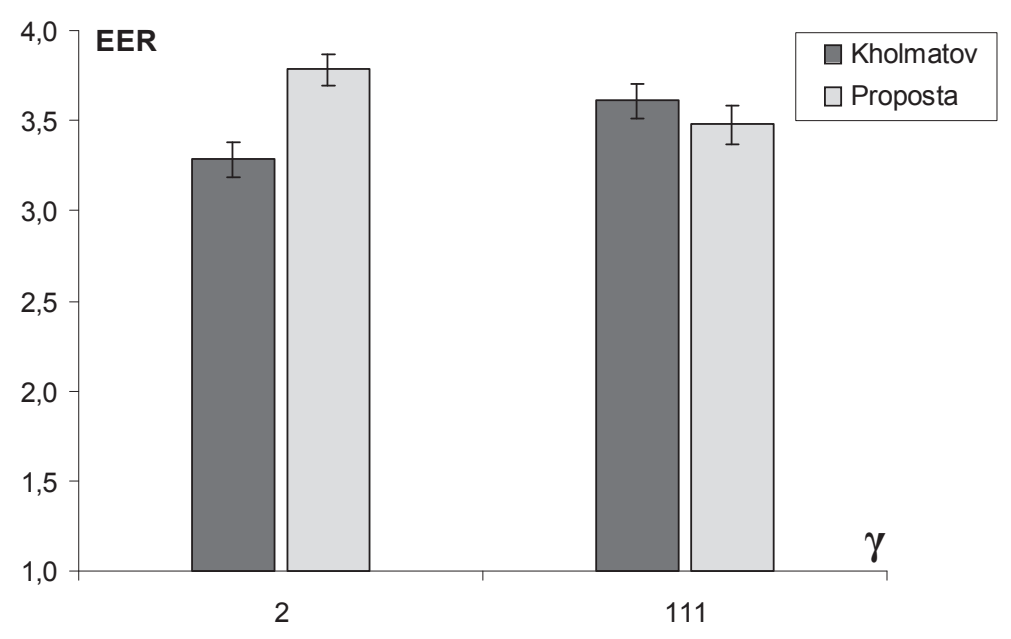

Figura 9. Médias de erros para os dois melhores valores de $y$

É possível notar que a representação de assinaturas proposta neste trabalho é comparável à original (Kholmatov \& Yanikoglu (2004)). Observe que embora não tenha sido superior, os desvios padrões foram baixos.

Além disso, as taxas de erros obtidas com as propostas aqui apresentadas são inferiores às taxas apresentadas por outros trabalhos. O que mostrou serem promissoras tanto a escolha do parâmetro $\theta$, calculado de forma dinâmica, quanto a representação da assinatura, levando em consideração sua curvatura.

Ao serem comparados os resultados obtidos na Seção 5.2, percebe-se que os valores de EER ora obtidos são menores. A razão provável para este comportamento corresponde à mudança do usuário utilizado no treinamento.

Finalmente, destaca-se que resultados obtidos com a implementação do sistema proposto por Kholmatov com sua representação de atributos da assinatura, $\left(\Delta_{x}, \Delta_{y}\right)$, foram próximos ao valor divulgado na competição de assinaturas, sit (2004), (2,8\%), onde este obteve o primeiro lugar.

\section{Conclusão}

O aumento constante do número de falsificações de assinaturas e os problemas acarretados pelas mesmas nos inúmeros sistemas que dependem da garantia da autenticidade têm criado demanda por formas automáticas de autenticação. Em especial 
a autenticação automática de assinaturas por meio de sistemas computacionais tem atraído a atenção de pesquisadores da área de visão computacional e reconhecimento de padrões, dado que é factível verificar se uma dada assinatura é ou não reconhecida como tendo os padrões de uma assinatura previamente armazenada, vinculada a uma pessoa, combinadas com técnicas da visão computacional. Com a automatização objetiva-se maior rapidez para o processo e com precisão aceitável na avaliação da autenticidade (ou a falsidade) de uma assinatura.

Em grande parte dos bancos o setor de compensação de cheques é operado por funcionários não-especialistas, geralmente um operador de caixa. Estes funcionários, com apenas algum treinamento prévio, analisam se a assinatura de um cheque, por exemplo, é pertencente ao correntista, ou não, comparando a mesma com a forma de um exemplar da assinatura cadastrada no sistema, podendo ou não aceitar a compensação do cheque.

Os sistemas de autenticação de assinaturas atuais ainda apresentam um desempenho insatisfatório em dois contextos: quando o sistema autentica assinaturas capturadas de forma offline, e/ou quando as falsificações fornecidas para testes são falsificações classificadas como habilidosas.

Neste artigo investigamos alguns problemas encontrados na autenticação de assinaturas obtidas online e assinaturas classificadas como falsificações habilidosas.

Numa etapa inicial, foi desenvolvido um estudo para avaliar o comportamento do algoritmo de programação dinâmica Dynamic Time Warping (DTW), aplicado à autenticação de assinaturas online. Para isso, foi verificada qual a influência individual dos principais parâmetros deste algoritmo no processo de autenticação de assinaturas, variando-os sobre uma ampla faixa de valores. Com o resultado das médias dos erros obtidos na classificação das assinaturas, para cada combinação dos parâmetros, pode-se avaliar a influência de cada um deles, dentro do contexto proposto.

Foi possível verificar como os parâmetros influenciam o alinhamento e o cálculo das distâncias entre duas sequências de dados representando as assinaturas, bem como estes influenciam a classificação de uma assinatura como sendo genuína ou falsa. Nos experimentos realizados foi observado que o parâmetro $\theta$ influencia o resultado dos erros obtidos com maior peso do que o parâmetro $\gamma$, e que um valor de $\theta$ muito alto tende a aumentar o número de erros.

Também foi identificado, a partir dos experimentos, que é provável a existência de um $\theta$ ótimo, para cada base de dados. Inferiu-se que, apesar dos resultados bons obtidos na classificação utilizando-se o $\theta$ ótimo para a base de assinaturas disponível, era improvável que os mesmos valores atribuídos para $\gamma$ e $\theta$ funcionassem de mesma forma em uma outra base de dados. Para garantir isto, seria necessário testá-los de forma exaustiva, o que é inviável na prática. Assim, formulou-se uma proposta para obter um $\theta$ subótimo, para aplicação prática do DTW, utilizando dados das próprias assinaturas sob comparação. Os experimentos com esta forma do cálculo de $\theta$ apresentaram resultados satisfatórios, podendo ser considerada uma contribuição à formulação do DTW original, que não expõe concretamente como este parâmetro deva ser calculado.

Foi explorada a possibilidade de se melhorar o desempenho do processo de autenticação aprimorando a forma de representação das assinaturas. Assim, investigou- 
se a utilização da informação da curvatura, a partir de vetores normais em cada ponto obtido pelo tablet. Para avaliar esta proposta e compará-la com a forma proposta no trabalho utilizado como base, o sistema descrito em Kholmatov \& Yanikoglu (2004, 2005) foi implementado para as duas versões de representação. Nesta implementação foi também empregada a proposta para o cálculo de $\theta$ desenvolvida na etapa anterior do estudo. A base de assinaturas disponível foi utilizada para avaliar e comparar o sistema do trabalho de base com o sistema contendo as modificações propostas.

Os resultados obtidos pela nova representação de assinaturas não foram consistentemente superiores à proposta por Kholmatov em Kholmatov \& Yanikoglu (2004, 2005), mas podem ser considerados comparáveis, de uma maneira geral, e superiores em alguns casos. Isto mostrou o traçado de um caminho promissor para melhores resultados. Observa-se que o sistema utilizado como base de comparação foi vencedor da competição First International Signature Verification Competition.

Os estudos desenvolvidos apontaram também caminhos para continuação das pesquisas. Inicialmente, podem ser analisadas formas de melhorar o cálculo do parâmetro $\theta$, pois é desejada a diminuição das taxas de erro em relação a este parâmetro. Além disto, apesar de não ter sido observada grande influência nas taxas de erro nos experimentos, é também interessante estudar uma forma para o cálculo do parâmetro $\gamma$ dinamicamente. Uma possível combinação dinâmica desses parâmetros pode ser decisiva para a obtenção de bons resultados, e assim, tentar diminuir o número de falsos positivos e negativos ocorridos no sistema.

Outras possibilidades de investigação são avaliar outras formas para representar a curvatura da assinatura de maneira a abranger suas características intra-classe, bem como diversificar a utilização de métodos e técnicas da área de visão computacional que possam auxiliar na distinção de uma assinatura falsificada das autênticas.

Agradecimento. Os autores gostariam de manifestar seus agradecimentos à CAPES e à Faperj pelo apoio financeiro concedido à presente pesquisa.

\section{Referências}

Svc 2004: First international signature verification competition. 2004.

A. Kholmatov, \& Yanikoglu, B., Susig: An on-line signature database, associated protocols and benchmark results. Pattern Analysis and Applications, 8(2):47-68, 2008.

Duda, R.O.; Hart, P.E. \& Stork, D.G., Pattern Classification. Second edition. Wiley, John \& Sons, Incorporated, 1999.

Garcia-Salicetti, S.; Houmani, N.; Ly-Van, B.; Dorizzi, B.; Alonso-Fernandez, F.; Fierrez, J.; Ortega-Garcia, J.; Vielhauer, C. \& Scheidat,T., Guide to Biometric Reference Systems and Performance Evaluation, Springer-Verlag. p. 125-165.

Johnson, R.A. \& Wichern, D.W., Applied Multivariate Statistical Analysis. Segunda Edição. New Jersey: Prentice Hall, 1982.

Kholmatov, A. \& Yanikoglu, B., Biometric authentication using online signatures. In: Rothlauf, F. \& Konstantinos, G., (Eds.). Proceedings International Symposium on 
Computer and Information Sciences (IS- CIS). Washington, USA: Springer LNCS3280, p. 373-380. 2004.

Kholmatov, A. \& Yanikoglu, B., Identity authentication using improved online signature veri_cation method. Pattern Recognition Letters, 26(2):2400-2408, 2005.

Krawczyk, S., User Authentication Using On-Line Signature And Speech. Dissertação de Mestrado, Department of Computer Science and Engineering, Michigan State University, 2005.

Wollmer, M.; Al-Hames, M.; Eyben, F.; Schuller, B. \& Rigoll, G., A multidimensional dynamic time warping algorithm for efficient multimodal fusion of asynchronous data streams. Neurocomputing (NEUCOM), 73(1-3):366-380, 2009. 